\title{
Radiometric normalization of high spatial resolution multi-temporal imagery: A comparison between a relative method and atmospheric correction
}

\author{
M. El Hajj*a ${ }^{*}$ M. Rumeau ${ }^{\mathrm{a}}$, A. Bégué ${ }^{\mathrm{a}}$, O. Hagolle ${ }^{\mathrm{b}}$, G. Dedieu ${ }^{\mathrm{b}}$ \\ ${ }^{a}$ UMR TETIS CIRAD-Cemagref-ENGREF, Remote Sensing Centre in Languedoc Roussillon, 500 \\ rue J.F. Breton 34093 Montpellier Cedex 5, France; \\ ${ }^{\mathrm{b}}$ Cesbio/CNES, 18 avenue E. Belin 31401 Toulouse Cedex 9, France.
}

\begin{abstract}
Radiometric normalization is a vital stage in the pre-processing of multi-temporal imagery. It aims to insure a reliable exploitation of images acquired under different imaging conditions. In this study, we investigate whether a relative normalization can replace atmospheric correction. The investigation was done using a time series of eighteen SPOT 5 images acquired over Reunion Island and intended to be used for sugarcane monitoring. An automatic method for relative normalization is introduced, and its results are compared to atmospherically corrected data. The relative method is based on the reflectances of invariant targets (IT) that are selected automatically. The atmospheric correction is carried out by the $6 \mathrm{~S}$ code. The comparison was performed a) by using a set of manually selected invariant targets (MSIT), and b) by assessing the NDVI behavior of a set of sugarcane fields. An excellent correlation is obtained between relatively and atmospherically corrected data: the coefficient of determination $\left(\mathrm{R}^{2}\right)$ is higher than 0.96 for all spectral bands and for the NDVI. Moreover, a comparable impact is observed on the temporal profiles of MSIT and on the NDVI trajectories of sugarcane field.
\end{abstract}

Keywords: Radiometric normalization, SPOT 5, Sugarcane, Time series, Invariant targets, multi-temporal imagery, 6S, Reunion Island

\section{INTRODUCTION}

Time series of satellite images acquired at high spatial and temporal resolutions constitute an important source of information for change detection and trend analysis. The dynamics of the radiometric signals and the image-derived indices are particularly interesting for agriculture monitoring, especially for the cartography of the interventions (sowing, harvest, irrigation,...), for the detection of growth anomalies, and for yield predictions.

Since multi-temporal images are often acquired by different sensors under different atmospheric conditions and at different solar illumination and view-angles, a radiometric normalization phase is required to remove radiometric distortions and to make the images comparable.

The atmospheric correction is usually carried out by a radiative transfer code that uses the atmosphere components which are measured simultaneously with image acquisitions. Several operational algorithms have been developed, among them we can quote: Modtran2 ${ }^{(1)}, 5 \mathrm{~S}^{(2)}, \mathrm{SMAC}^{(3)}$ and $6 \mathrm{~S}^{(4)}$.

Another way to correct the radiometric distortion due to the atmospheric effects is relative normalization. It does not require atmospheric data, and moreover, it corrects the deformation of the radiometry caused by directional effects: one image among the time series is used as a reference, and the radiometric properties of the other images are adjusted to match this reference. Many methods have been proposed ${ }^{(5-10)}$. They proceed under the assumption that the relationship between the TOA radiances recorded at two different times from regions of constant reflectance is spatially homogeneous and can be approximated by linear functions.

In this study, we introduce an automatic method for relative radiometric normalization based on invariant targets (IT). Since atmospheric data are not always available to do absolute radiometric correction for multi-temporal images, we investigate whether our relative normalization method can substitute the use of the atmospheric correction code $6 \mathrm{~S}$. 


\section{DATA SET DESCRIPTION}

\subsection{Study site}

The study site is Reunion Island. It is a small territory (ca. $2512 \mathrm{~km} 2)$ located in the Indian Ocean $\left(21^{\circ} 7^{\prime}\right.$ to $19^{\circ} 40^{\prime} \mathrm{S}, 55^{\circ}$ $13^{\prime}$ to $61^{\circ} 13^{\prime} \mathrm{E}$ ), at the north-east of Madagascar (Fig.1). Situated in a tropical zone, the year is divided into two seasons: the humid season from November to April, and the fresh season from May to October. The island is highly mountainous. There are smooth slopes on the coastal zones, which increase quickly toward the centre of the island. The centre is made of three cirques which give a very contrasted relief.

Sugarcane is the main culture in Reunion Island. It is cultivated along the coast on 26,500 ha (Source: DDAF 2004). Most of the growers are smallholders, and the average size of sugarcane fields is about 0.8 ha. In the wet north-eastern part of the island, sugarcane is rain fed, while in the drier south-western part it is irrigated.

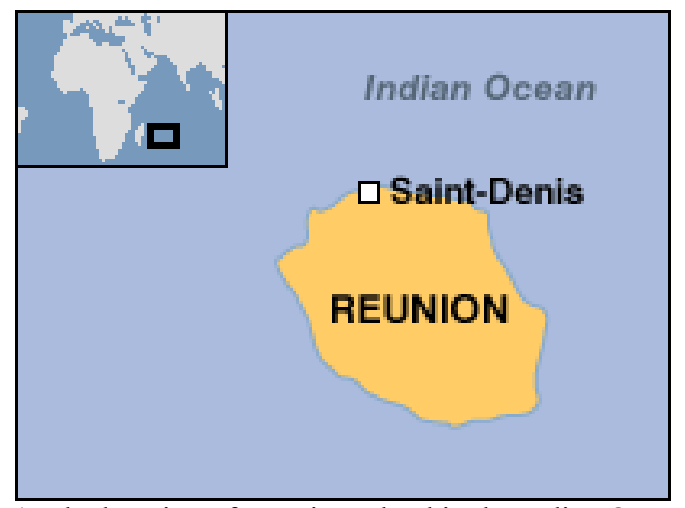

Fig. 1. The location of Reunion Island in the Indian Ocean.

\subsection{Data set}

The data set used in this study consists of eighteen SPOT 5 images acquired over Reunion Island. Both SPOT 5 instruments HRG1 and HRG2 acquire radiation in four spectral bands with high spatial resolution: 10x10m in Green, Red and NIR (Near Infra-Red) bands, and 20x20m in SWIR (Short Wave Infra-Red) band.

The images belong to the KALIDEOS database set up by the CNES ${ }^{(11)}$. All images are ortho-rectified and provided with two levels of radiometric correction: Top Of Atmosphere (TOA), and Top Of Canopy (TOC). Table 1 shows the characteristics of the time series, as well as the atmospheric data recorded at the acquisition dates. As it can be seen, the geometrical conditions of imaging and the atmospheric characteristics vary remarkably from a date to another.

On the other hand, cloud mask and saturated-pixels mask were available for each image. Moreover, a map of sugarcanecultivated fields was on hand.

Table 1. Characteristics of the imaging and atmospheric conditions of the time series.

\begin{tabular}{|c|c|c|c|c|c|c|c|c|}
\hline Dates & $\begin{array}{c}\text { SPOT } 5 \\
\text { Instrument }\end{array}$ & $\begin{array}{c}\text { Incidence angle } \\
\left.\text { (in }{ }^{\circ}\right) \\
(\text { Right }=-)\end{array}$ & $\begin{array}{c}\text { Solar elevation } \\
\text { (in }{ }^{\circ} \text { ) }\end{array}$ & $\begin{array}{l}\text { Phase angle } \\
\text { (in }{ }^{\circ} \text { ) }\end{array}$ & $\begin{array}{c}\text { Pressure } \\
\text { (mbar) }\end{array}$ & $\begin{array}{c}\mathrm{H}_{2} \text { Oatm } \\
\left(\mathrm{g} \mathrm{cm}^{-2}\right)\end{array}$ & $\begin{array}{c}\text { Ozone } \\
\text { (cm.at } \\
\text { m) }\end{array}$ & $\tau_{550}$ \\
\hline $01 / 10 / 2003$ & HRG 2 & $-04,65$ & 64,10 & 21,28 & 1014 & 2,783 & 0,264 & 0,538 \\
\hline $02 / 26 / 2003$ & HRG 1 & $-11,94$ & 58,54 & 22,07 & 1013 & 5,469 & 0,259 & 0,322 \\
\hline $04 / 24 / 2003$ & HRG 1 & $-04,39$ & 48,02 & 40,23 & 1017 & 4,24 & 0,253 & 0,246 \\
\hline $05 / 04 / 2003$ & HRG 1 & 10,90 & 46,80 & 47,99 & 1015 & 2,649 & 0,252 & 0,262 \\
\hline $07 / 21 / 2003$ & HRG 1 & 10,58 & 41,20 & 53,13 & 1022 & 2,332 & 0,263 & 0,112 \\
\hline $08 / 21 / 2003$ & HRG 1 & 18,17 & 48,90 & 51,00 & 1024 & 2,151 & 0,272 & 0,273 \\
\hline
\end{tabular}




\begin{tabular}{|l|l|l|l|l|l|l|l|l|}
\hline $09 / 01 / 2003$ & HRG 1 & $-04,42$ & 50,63 & 37,31 & 1026 & 1,954 & 0,276 & 0,277 \\
$10 / 08 / 2003$ & HRG 1 & $-25,95$ & 60,40 & 19,75 & 1018 & 2,671 & 0,297 & 0,432 \\
$12 / 19 / 2003$ & HRG 1 & $-02,90$ & 67,20 & 19,90 & 1017 & 3,093 & 0,272 & 0,357 \\
$03 / 17 / 2004$ & HRG 2 & -19.10 & 54.2 & 25.24 & 1014 & 2,761 & 0,255 & 0,176 \\
$04 / 11 / 2004$ & HRG 1 & +17.95 & 52.45 & 48.41 & 1014 & 4,793 & 0,252 & 0,26 \\
$05 / 13 / 2004$ & HRG 1 & -11.80 & 42.90 & 43.86 & 1018 & 1,986 & 0,246 & 0,22 \\
$06 / 18 / 2004$ & HRG 2 & +03.25 & 39.10 & 51.95 & 1024 & 2,357 & 0,237 & 0,11 \\
$07 / 09 / 2004$ & HRG 1 & -04.73 & 38.83 & 49.70 & 1020 & 2,674 & 0,242 & 0,221 \\
$08 / 19 / 2004$ & HRG 1 & +17.96 & 48.50 & 51.24 & 1027 & 2,126 & 0,254 & 0,197 \\
$10 / 26 / 2004$ & HRG 2 & +03.30 & 67.90 & 24.94 & 1018 & 2,946 & 0,275 & 0,329 \\
$11 / 06 / 2004$ & HRG 1 & -19.16 & 66.63 & 09.07 & 1021 & 2,541 & 0,286 & 0,351 \\
$12 / 07 / 2004$ & HRG 1 & -12.28 & 66.65 & 11.19 & 1021 & 1,962 & 0,276 & 0,355 \\
\hline
\end{tabular}

\section{METHODS}

\subsection{Relative radiometric normalization}

The proposed method for relative radiometric normalization is a regression-based approach that attempts to uniformly minimize the effects of changing atmospheric and geometric conditions relatively to a reference. This method consists in three main steps: the choice of a reference image, the IT selection, and the regression coefficients calculation.

\subsubsection{Reference image}

Among the images in the time series, one must be chosen to be the reference to which all the other scenes will be related. This image must be the least cloud-contaminated, time-wise adequate for the application, and must have a good spectral dynamic range. The reference image we chose for our normalization is the one acquired in 13 May 2004 (c.f. Fig. 2).

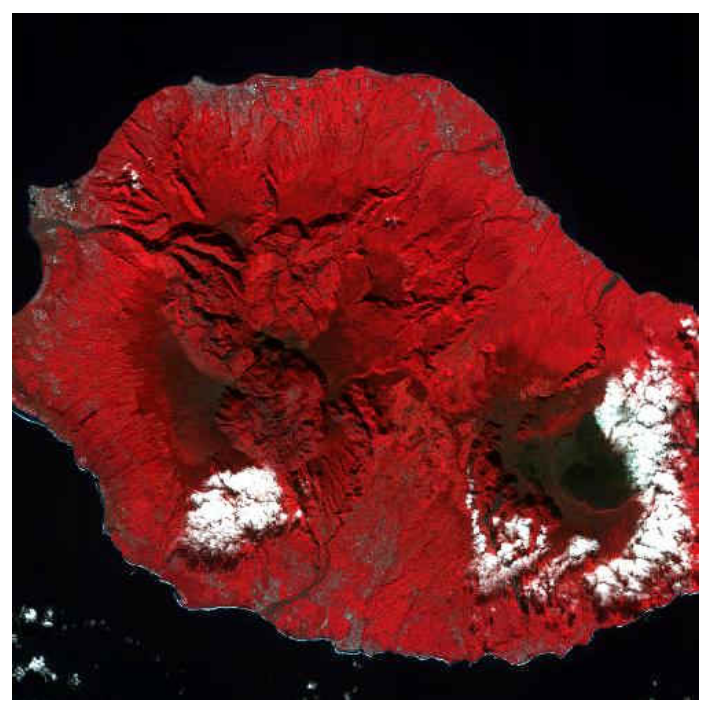

Fig. 2. The color composite (Red: band-3; Green: band-2; Blue: band-1) image of the reference selected for the relative radiometric normalization. 


\subsubsection{Selection of IT}

We developed an automatic technique for IT selection. The motive was to make the selection process independent from the effects of subjective performance, and to obtain a sufficient number of IT that cover a large spectral range.

Furthermore, we manually selected another set of IT in order to validate the automatic selection technique. This set of manually selected IT (MSIT) consists of 70 features of $20 \mathrm{~m} * 20 \mathrm{~m}$ spread on the whole Island surface and covering a large spectral range. A subset $V$ of the MSIT was also used in the in the comparison performed between the relatively and the atmospherically corrected data. In this sub-section we will describe the automatic IT selection technique, and the results of its validation process will be shown later.

The flowchart of the automatic IT selection technique is illustrated in Fig. 3. First, we calculate for each image in the time series a corresponding difference image, and this, by a pixel-based subtraction with the reference image in each spectral band. Then, by using the cloud masks, we flag, in each difference image, the pixels related to cloud in either the original or the reference images. After that, pixels in the difference images resulting from saturated pixels, in at least one of the four bands of the original images, are also flagged using the saturation masks. Then, in order to reduce the number of pixels related to volatile areas, we use the map of the sugarcane fields as a mask; so pixels of sugarcane, that constitute $60 \%$ of the cultivated areas in Reunion Island, are flagged. Next, since we are not interested in having IT in the ocean, we use the site mask to flag ocean pixels. Afterwards, for each date, the selection of IT is performed by using the histogram of the unflagged pixels of the difference image:

Actually, the histogram shape of each band of the difference image depends on the type of changes that happen between the image date and the reference date. Each spectral band is sensitive to different sorts of change: for instance, a soil type change can cause a significant modification of pixel value in one spectral band but not in the others. In all bands, the pixels which have relatively slight changes will be clustered around the peaks of the difference image histograms. This means that the majority of the unflagged pixels is considered as targets with no or nearly no change. The difference values corresponding to these pixels differ from zero because of the change in imaging conditions from a date to another. The centre of these clusters does not necessarily correspond to histogram-bands mean positions because of unequal frequencies of changes with equal magnitudes and different signs. The rest of the histogram belongs to the pixels with real land changes. These pixels may have been affected by different imaging conditions but their little effect compared to real change is negligible.

Histograms, in the four spectral bands, are then shifted so that the difference values assigned to the majorities (peaks) are brought back to zero. Finally, pixels which the difference values, in the four bands simultaneously, are less than seven percent of the majority standard deviation $(7 \% * \sigma)$ are considered as IT. The average percentage (over all images) of the selected IT according to the Island number of pixels is $0.044 \%$.

\subsubsection{Regression coefficients calculation}

For selected IT, mean reflectance values in the four spectral bands were extracted from the images, and mean NDVI values were calculated. Using these values, linear regressions of the form $y=a . x+b$ were established for each band and for the NDVI, where $y$ is the reference image and $x$ are the other images in turn. The regressions were then applied to the images to perform the relative normalization.

\subsubsection{Validation of the automatic selection of IT}

In order to validate the technique of automatic selection of IT, a subset $U$ of the MSIT was used to establish, for each date, other linear regressions $y=a^{\prime} \cdot x+b^{\prime}$, where, as before, $y$ is the reference image and $x$ are the other images in turn. These regressions and the ones obtained by the automatic selection technique were applied on the other subset $V$ of the MSIT. Fig. 4 shows that the correlation between the normalization resulting from the two IT selection approaches is very strong ( $\mathrm{R}^{2}>0.98$ for all spectral bands), and moreover, the slope values of regressions are very close to unity and the intercepts are near zero. Consequently, we consider that the proposed automatic selection technique of IT is successful and it is an excellent alternative to the time-consuming manual IT selection. 


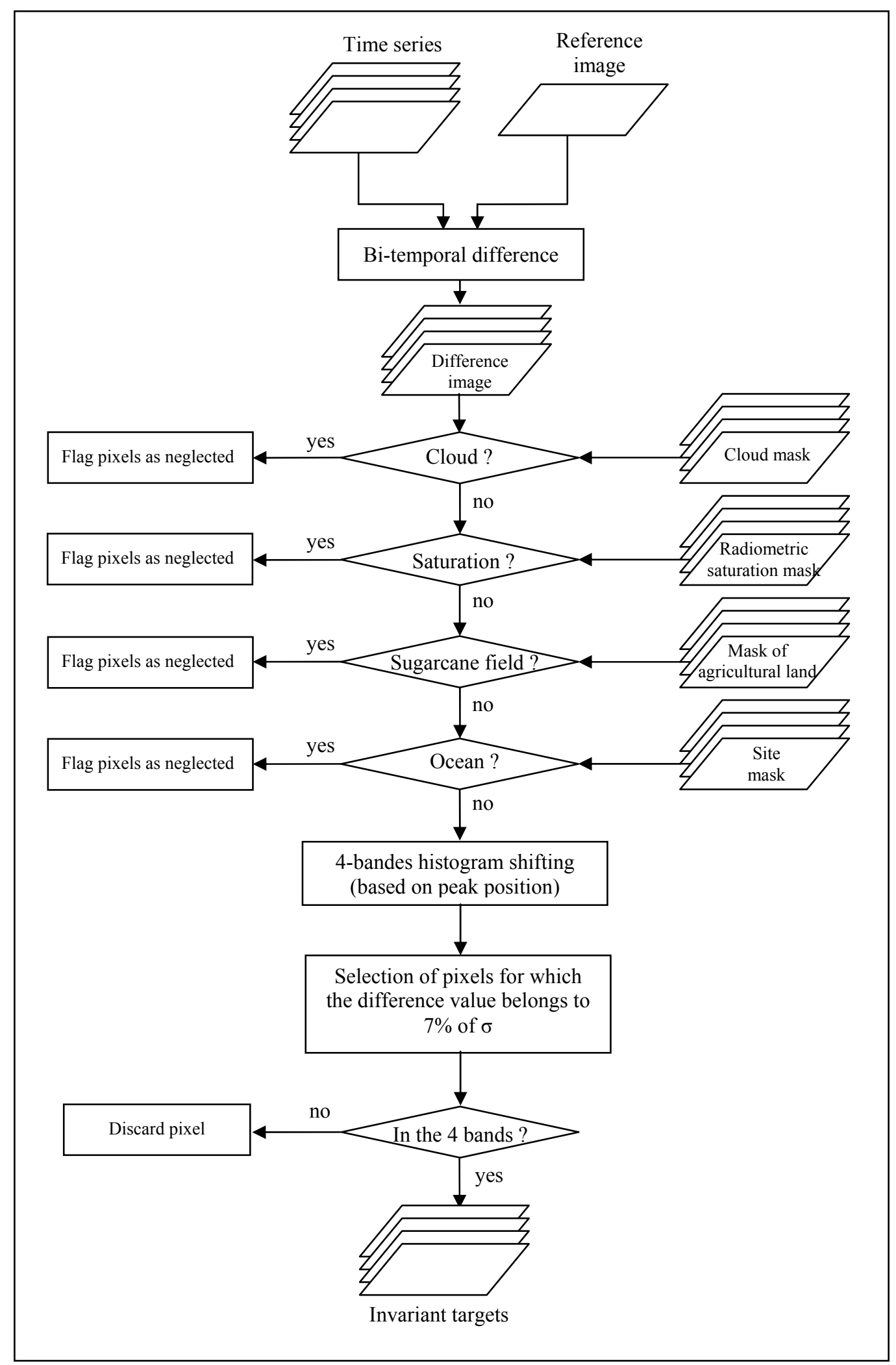

Fig. 3. The flowchart of the automatic IT selection technique. 


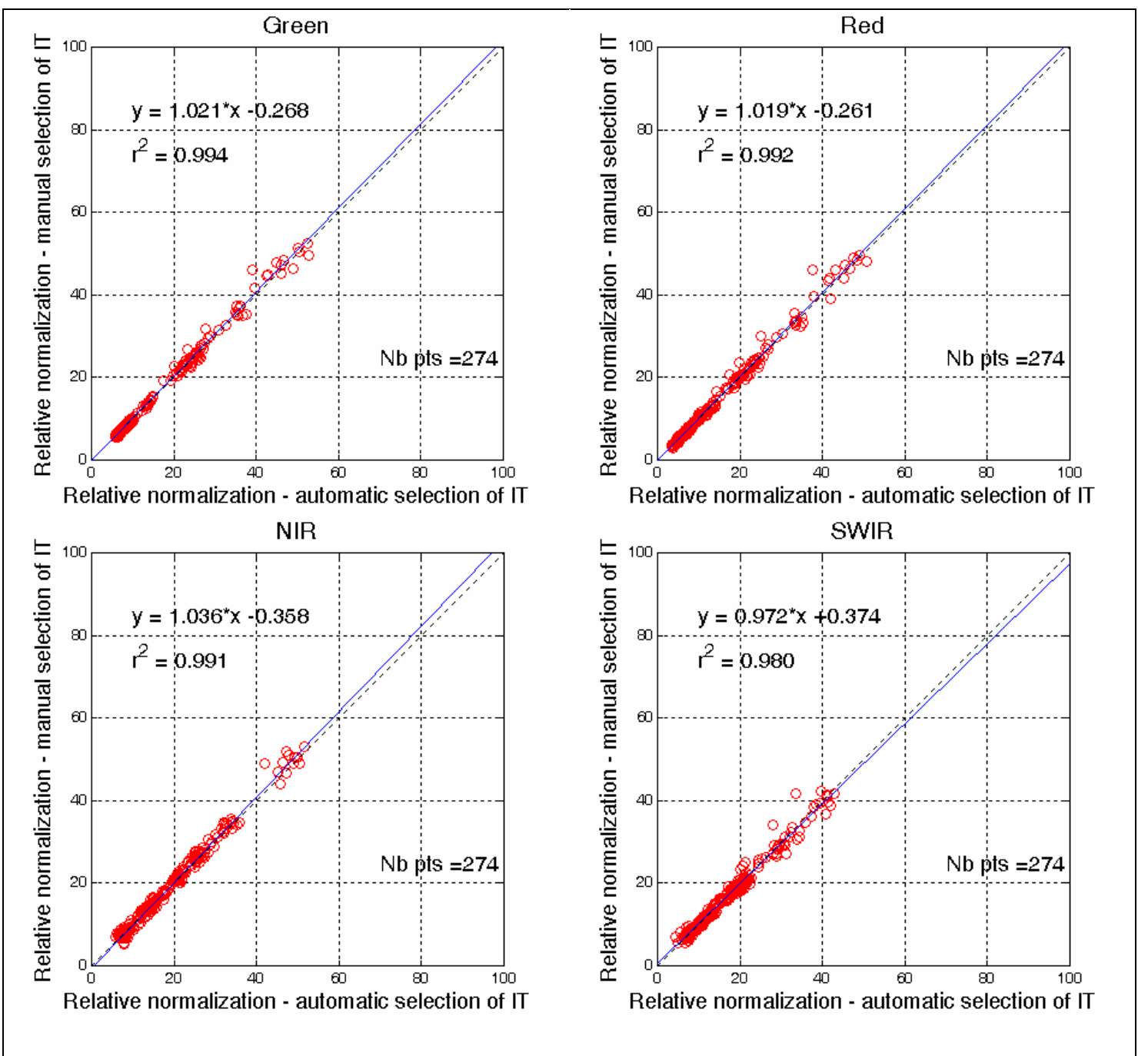

Fig. 4. Regressions obtained in each band for the validation of the automatic selection technique of IT: the x-axis corresponds to the normalized values obtained by the automatic selection technique, and the y-axis to the normalized values obtained by the subjective selection of IT.

\subsection{Atmospheric correction}

Atmospheric correction is an old and currently addressed issue and many codes exist: Lowtran 7, Modtran 2, 5S, SMAC, $6 \mathrm{~S}$, etc... The aim of these codes is to retrieve the TOC reflectance from the TOA one derived from radiance measured by sensors. We will not detail here how these models work, but we only mention that the radiative transfer code used to produce the TOC images used in this study is the $6 \mathrm{~S}$ code. $6 \mathrm{~S}$ input variables are: the view and solar angles, the atmosphere composition (optical thickness, water vapor content, atmospheric pressure, ozone content...), and the target slope and its environment. The main difficulty in realizing the atmospheric correction was to obtain accurate measurement of the aerosol optical properties. Since no in-situ measurements were available, the aerosol optical thickness (AOT) values $\left(\tau_{550}\right)$ were derived from SeaWiFS Level 3 data: for each image, the AOT used is the average of the AOT values measured around the Island above the ocean. The used water vapor content values stem from climatologic database. 


\section{RESULTS}

The comparison between the performances of the relative radiometric normalization and the atmospheric correction code was carried out according to two axes:

- $\quad$ the first concerns the impact of each method on the reflectances of MSIT,

- $\quad$ and the second relates to the influence of the methods on the mean NDVI values calculated at sugarcane field scale.

\subsection{Comparison using MSIT}

As mentioned before, a subset $V$ of the MSIT was employed in the comparison between the relatively and the atmospherically corrected data. This subset comprises twenty homogenous targets of $20 * 20 \mathrm{~m}^{2}$ : large buildings, dense forests, volcanic lavas, bare soils, airport tracks, tennis court, etc ... They cover a large spectral range: Fig. 5 shows the distribution of the mean values of the TOA reflectance calculated over the acquisition dates for each target in $V$.

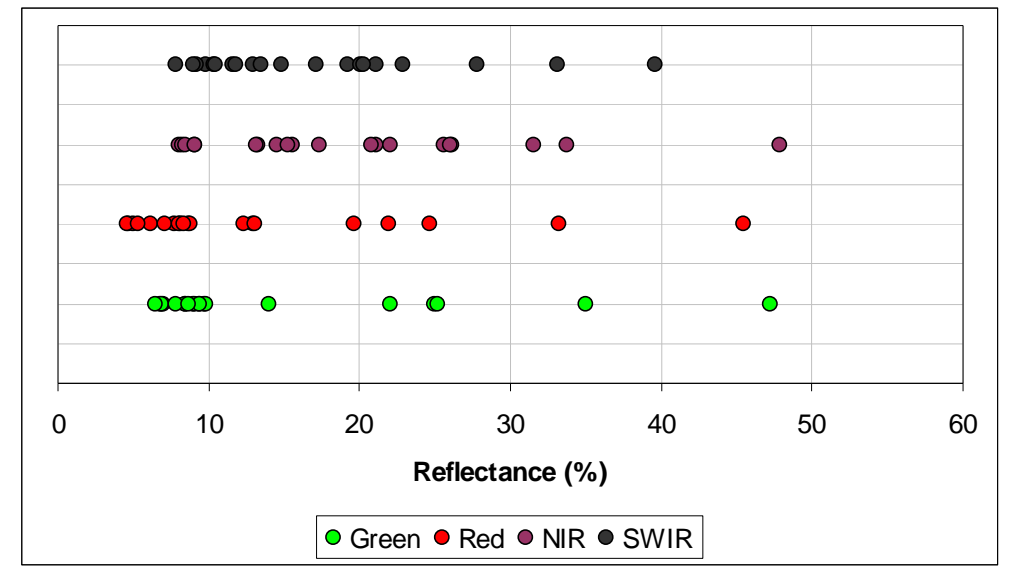

Fig. 5. Mean reflectance values (over time) of the MSIT belonging to the subset $V$.

W e extracted, for each MSIT, the mean values of the TOC reflectances at each date. On the other band, at each date also, the relatively normalized images were used to obtain the relatively normalized reflectance values of each MSIT. In Fig. 6 we plot the relatively normalized reflectance of MSIT as a function of its atmospherically corrected reflectance (TOC), and this for all spectral bands. An excellent correlation is found in all bands: $0.992,0.994,0.983$ and 0.960 in Green Red NIR and SWIR respectively. The slope values are very close to one, and the intercept values are less than 4.6.

Furthermore, we were interested in investigating the impact of each method on the temporal behavior of the MSIT reflectances. For that, we calculated for each MSIT the standard deviation of its profile over the eighteen acquisition dates: a) before normalization (TOA reflectances), b) after relative normalization, and c) after atmospheric correction (TOC reflectances). Due to lack of space, we only show the average and the maximum values of the standard deviation obtained at each level in each band (Table 2). When looking at the average standard deviations, we notice that both the relative and the atmospheric correction methods smooth the temporal profiles of the MSIT reflectance (for all spectral bands). However, the relative radiometric normalization smooth better these profiles, and moreover, it decreases also the maximum value of the standard deviations which is not the case for the atmospheric correction. This might be due to the fact that the relative normalization corrects also the effects of the directionality. Consequently, we can say that the proposed relative normalization method effectively minimizes the radiometric distortion present in the time series of images.

\subsection{Comparison using sugarcane fields}

Since the final aim of this project is to monitor sugarcane using the multi-temporal images, it was necessary to assess the impact of each method on the NDVI values calculated at sugarcane field scale. Actually, the NDVI profile is a very good tool for the yield prediction and the harvest detection process. 
Eighteen fields were chosen to realize the comparison. The average size of these fields was 4.3 ha. For each field, we calculated the mean NDVI value at each date using: a) the relatively normalized images and b) the TOC images.

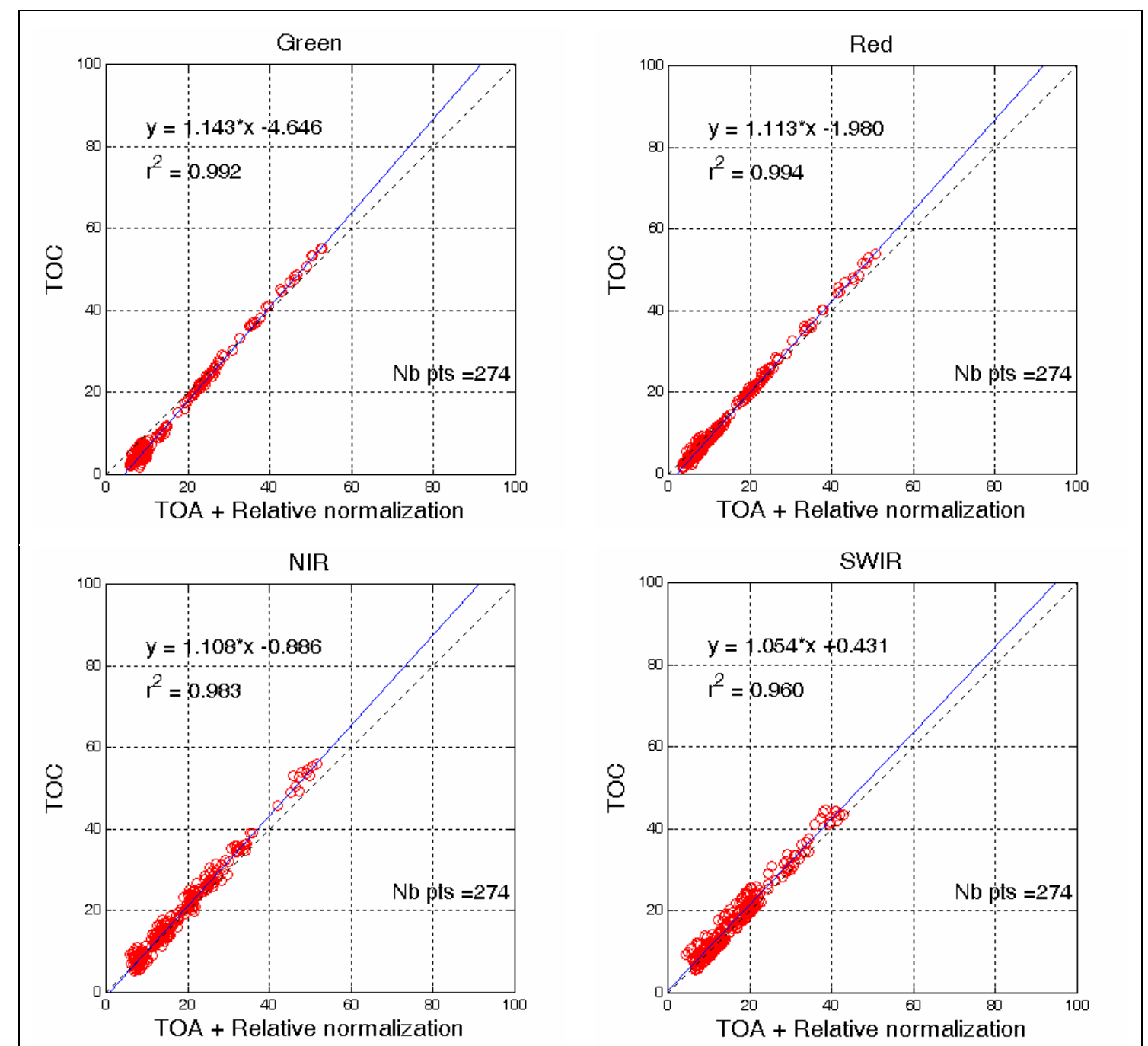

Fig. 6. Comparison between the MSIT reflectances corrected by $6 \mathrm{~S}$ (TOC) and those corrected by the relative radiometric normalization.

\begin{tabular}{|c|c|c|c|c|c|c|c|c|c|c|c|c|}
\hline & \multicolumn{4}{|c|}{ TOA } & \multicolumn{4}{|c|}{ TOA + Relative normalization } & \multicolumn{4}{|c|}{$\begin{array}{c}\text { TOA + Atmospheric correction } \\
\text { (TOC) }\end{array}$} \\
\hline & G & $\mathrm{R}$ & NIR & SWIR & G & $\mathrm{R}$ & NIR & SWIR & $\mathrm{G}$ & $\mathrm{R}$ & NIR & SWIR \\
\hline Average STD & 1.34 & 1.59 & 1.80 & 2.08 & 0.88 & 1.10 & 1.25 & 1.25 & 1.23 & 1.46 & 1.69 & 1.98 \\
\hline Maximum STD & 3.43 & 3.54 & 2.95 & 4.21 & 3.04 & 3.21 & 2.63 & 2.59 & 3.51 & 3.57 & 2.86 & 4.08 \\
\hline
\end{tabular}

Table 2. Average and maximum values of the standard deviation (STD) of the MSIT reflectances: before radiometric normalization (TOA), after relative radiometric normalization, and after atmospheric correction (TOC). 
Fig. 7.a shows the excellent correlation $\left(\mathrm{R}^{2}=0.958\right)$ obtained between the NDVI values calculated for all dates and all fields. The slope and intercept values are, one more time, close to one and zero respectively.

On the other hand, we compared the relative evolution of NDVI from one date to another resulting from each method. For that, for each field, we calculated the NDVI slope values at each consecutive couple of dates. Fig. 7.b figures out the comparison of NDVI slopes. Considering the strong correlation $\left(\mathrm{R}^{2}=0.979\right)$ and the low level of dispersion around the regression, we can consider that both correction methods lead to a very comparable temporal evolution of NDVI.

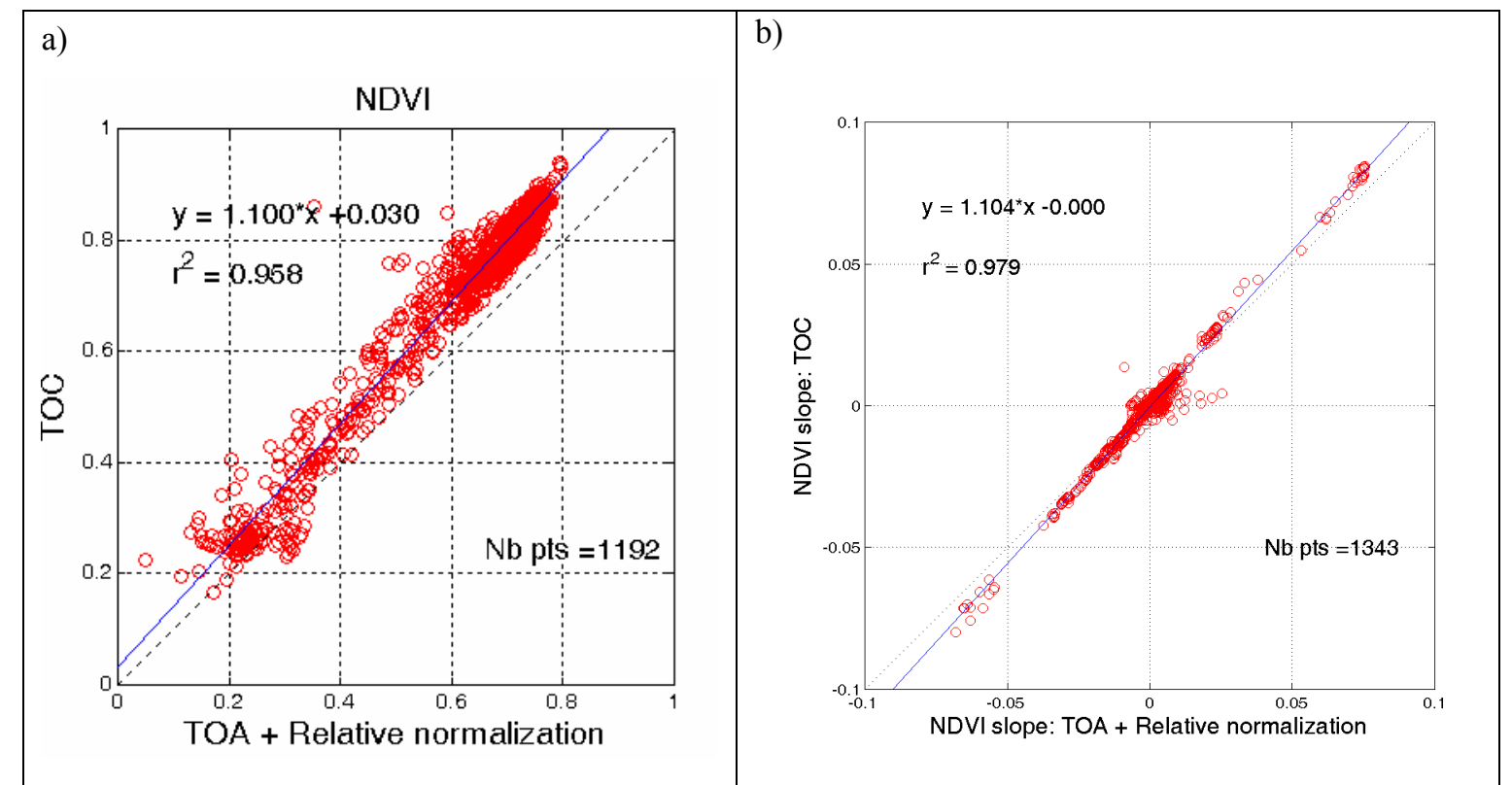

Fig. 7 . a) Comparison between NDVI values calculated at sugarcane field scale and corrected by 6S (TOC) and those corrected by the relative radiometric normalization. b) Comparison between NDVI slope values corrected by 6S (TOC) and those corrected by relative normalization (all fields)

\section{CONCLUSIONS}

This paper deals with the radiometric normalization of a SPOT 5 time series of images acquired over Reunion Island. A relative method of automatic radiometric normalization was presented and compared to the performance of an atmospheric correction carried out by the $6 \mathrm{~S}$ code. The relative normalization method is based on invariant targets reflectances. Since finding these targets is an important step in the relative normalization process, an automatic technique for IT selection was developed and validated.

The comparison between the relatively and the atmospherically corrected data was performed first by using manually selected invariant targets and second on sugarcane fields. The results demonstrated that the proposed relative normalization method is an excellent alternative to the atmospheric correction code $6 \mathrm{~S}$ : the correlation is strong in all spectral bands and the temporal profiles of NDVI at sugarcane field scale are very comparable.

\section{ACKNOWLEDGEMENT}

Mahmoud El Hajj is supported by a Cemagref/Région Languedoc Roussillon Ph.D. fellowship. Special thanks to Hélène de Boissezon (CNES) for her support. We also thank Thierry Rabaute (CS-SI) and Bruno Lafrance (CS-SI) for performing the geometric and the radiometric corrections of the images. 


\section{REFERENCES}

1. G. P. Anderson, J. H. Chetwynd, J.-M. Theriault, P. Acharya, A. Berk, D. C. Robertson, F. X. Kneizys, M. L. Hoke, L. W. Abreu and E. P. Shettle, "MODTRAN2: suitability for remote sensing", 1968, 514-525, Publ by Society of PhotoOptical Instrumentation Engineers, City, Year.

2. D. Tanre, "Description of a computer code to simulate the satellite signal in the solar spectrum: the 5S code," International Journal of Remote Sensing, 11, 659-668, (1990).

3. H. Rahman and G. Dedieu, "SMAC: a simplified method for the atmospheric correction of satellite measurements in the solar spectrum," International Journal of Remote Sensing, 15, 123-143, (1994).

4. E. F. Vermote, D. Tanre, J. L. Deuze, M. Herman and J. J. Morcrette, "Second simulation of the satellite signal in the solar spectrum, 6s: an overview," IEEE Transactions on Geoscience and Remote Sensing, 35, 675-686, (1997).

5. Y. Du, P. M. Teillet and J. Cihlar, "Radiometric normalization of multitemporal high-resolution satellite images with quality control for land cover change detection," Remote Sensing of Environment, 82, 123-134, (2002).

6. S. L. Furby and N. A. Campbell, "Calibrating images from different dates to 'like-value' digital counts," Remote Sensing of Environment, 77, 186-196, (2001).

7. F. G. Hall, D. E. Strebel, J. E. Nickeson and S. J. Goetz, "Radiometric rectification: toward a common radiometric response among multidate, multisensor images," Remote Sensing of Environment, 35, 11-27, (1991).

8. V. Houlès, M. El Hajj and A. Bégué, "Radiometric normalization of a Spot 4 and Spot 5 time series of images (ISLE-REUNION) for agriculture applications", 7p., City, Year.

9. M. S. Moran, R. D. Jackson, P. N. Slater and P. M. Teillet, "Evaluation of simplified procedures for retrieval of land surface reflectance factors from satellite sensor output," Remote Sensing of Environment, 41, 169-184, (1992).

10. J. R. Schott, C. Salvaggio and W. J. Volchok, "Radiometric scene normalization using pseudoinvariant features," Remote Sensing of Environment, 26, 1-16, (1988).

11. H. DeBoissezon and A. Sand, "Reference Remote Sensing Data Bases: Temporal series of calibrated and orthorectified satellite images for scientific use", City, Year. 